Article

\title{
Long Chain Fatty Acid Acylated Derivatives of Quercetin-3-O-Glucoside as Antioxidants to Prevent Lipid Oxidation
}

\author{
Sumudu N. Warnakulasuriya, Ziaullah and H. P. Vasantha Rupasinghe * \\ Department of Environmental Sciences, Faculty of Agriculture, Dalhousie University, Truro, \\ NS B2N 5E3, Canada; E-Mails: sumudunw@dal.ca (S.N.W.); Ziaullah.ziaullah@dal.ca (Z.) \\ * Author to whom correspondence should be addressed; E-Mail: vrupasinghe@dal.ca; \\ Tel.: +1-902-893-6623; Fax: +1-902-893-1404.
}

Received: 9 September 2014; in revised form: 16 October 2014 / Accepted: 27 October 2014 / Published: 6 November 2014

\begin{abstract}
Flavonoids have shown promise as natural plant-based antioxidants for protecting lipids from oxidation. It was hypothesized that their applications in lipophilic food systems can be further enhanced by esterification of flavonoids with fatty acids. Quercetin-3-O-glucoside (Q3G) was esterified individually with six selected long chain fatty acids: stearic acid (STA), oleic acid (OLA), linoleic acid (LNA), $\alpha$-linolenic acid (ALA), eicosapentaenoic acid (EPA) and decosahexaenoic acid (DHA), using Candida antarctica B lipase as the biocatalyst. The antioxidant activity of esterified flavonoids was evaluated using lipid oxidation model systems of poly-unsaturated fatty acids-rich fish oil and human low density lipoprotein (LDL), in vitro. In the oil-in-water emulsion, Q3G esters exhibited 50\% to 100\% inhibition in primary oxidation and $30 \%$ to $75 \%$ inhibition in secondary oxidation. In bulk oil, Q3G esters did not provide considerable protection from lipid oxidation; however, Q3G demonstrated more than 50\% inhibition in primary oxidation. EPA, DHA and ALA esters of Q3G showed significantly higher inhibition in $\mathrm{Cu}^{2+}$ - and peroxyl radical-induced LDL oxidation in comparison to Q3G.
\end{abstract}

Keywords: Q3G esters; fatty acids; antioxidant; lipid oxidation; LDL oxidation 


\section{Introduction}

There has been a strong interest in nutritional benefits of omega-3 (n-3) poly-unsaturated fatty acids (PUFA) containing dietary lipids due to their clinical benefits associated with prevention of chronic diseases, such as cardiovascular diseases and cancer [1,2]. However, foods rich in PUFA are highly susceptible to oxidation, leading to rancidity and nutritional loss [2,3]. The oxidation of dietary unsaturated fatty acids results in generation of advanced lipid oxidation end-products which are in part cytotoxic and genotoxic [4].

Lipid oxidation can occur at the cellular level, in the plasma membrane, as well as in low density lipoprotein (LDL)-cholesterols, due to the presence of cellular reactive oxygen species and is responsible for many pathological conditions [5]. The oxidation of PUFA within the forms of oxidized LDL or atherogenic LDL and therefore, is a causal factor for pathogenesis of atherosclerosis [6]. Habitual consumption of dietary antioxidants is one of the ways to retard the oxidative stress mediated chronic diseases [7]. Potential safety risks associated with the synthetic antioxidants has created an increasing market demand for natural antioxidants [3]. Many natural polyphenols including flavonoids are abundant mainly in plant foods, such as fruits and their products. However, lipophilicity of an antioxidant molecule is important for its effectiveness when incorporated into different food matrices [6].

The moderately hydrophilic nature of naturally occurring flavonoid glycosides, could become a limiting factor when incorporating them into more lipophilic food systems $[8,9]$. The structural modification of flavonoid molecules is one of the possible approaches to enhance their incorporation in lipophilic food products such as fish oil. The preparation of lipophilic derivatives of flavonoids using aliphatic molecules has shown greater miscibility in lipophilic systems [10,11]. Enzymatic acylation of natural polyphenols with long chain fatty acids has been suggested for improving the lipophilic nature of the glycosylated flavonoids [8,12-14]. The present study is aimed at evaluating the ability of six unsaturated, mono- and poly-unsaturated long chain fatty acid derivatives of Q3G in inhibiting oxidation of PUFA in lipid food model systems of oil-in-water emulsions and bulk oil and inhibition of $\mathrm{Cu}^{2+}$ - and peroxyl radical-induced oxidation of human LDL in vitro.

\section{Material and Methods}

\subsection{Chemicals and Supplies}

Ferric chloride, dimethyl sulfoxide, 2,2'-azobis(2-amidinopropane) dihydrochloride (AAPH), trichloroacetic acid (TCA), 2-thiobarbituric acid (TBA), butylated hydroxytoluene (BHT), molecular sieves and Candida antactica B lipase were obtained from Sigma-Aldrich (Oakville, ON, Canada). Acetic acid, hydrochloric acid, HPLC grade chloroform, and HPLC grade methanol were purchased from Fisher Scientific (Ottawa, ON, Canada). Ethanol anhydrous was obtained from Commercial Alcohols (Montreal, QC, Canada). Round bottom 96-well plates were purchased from Corning Incorporated (Edison, NY, USA). Quercetion-3-O-glucoside (Q3G) was obtained from Indofine Chemical Company (Hillsborough, NJ, USA). Bulk fish oil was a generous gift from BASF A/S (Malmparken 5, Ballerup, Denmark). The composition of the oil was $19.1 \%$ cis-monounsaturates and $33.7 \%$ cis-polyunsaturates ( $29.9 \% \omega-3$ polyunsaturated fatty acids, $3.7 \% \omega-6$ polyunsaturated fatty acids), $1.2 \%$ trans fatty acids, $25.6 \%$ saturated fatty acids, 5.6\% EPA, 22.9\% DHA by weight). LDL isolated from human plasma 
(in $150 \mathrm{mM} \mathrm{NaCl}, 0.01 \%$ EDTA, $\mathrm{pH}$ 7.4) was purchased from EMD Chemicals Inc. (Gibbstown, NJ, USA). Free fatty acids were purchased from Nu-Check-prep, Inc. (Waterville, MN, USA). All other chemicals were purchased from Fisher Scientific.

\subsection{Synthesis of Fatty Acid Acylated Derivatives of $Q 3 G$}

Synthesis of fatty acid esters of Q3G (phenolipids) was carried out through enzymatic esterification of Q3G separately with stearic acid (STA), oleic acid (OLA), linoleic acid (LNA), $\alpha$-linolenic acid (ALA), eicosapentaenoic acid (EPA) and decosahexaenoic acid (DHA) as acyl donors as previously described [14] (Figure 1). Briefly, Q3G (500 mg) and each acyl donor were added into a reaction vessel containing dried $3 \AA$ molecular sieves in a molar ratio of flavonoid:acyl donor 1:5. Anhydrous acetone was used as the solvent. The acylation was initiated by adding Candida antarctica B immobilised lipase $\left(2 \mathrm{~g}\right.$ ) as the biocatalyst. Then, the mixture was incubated at $45^{\circ} \mathrm{C}$ while stirring for approximately $48 \mathrm{~h}$ in a sand bath. Enzymatic conversion of the substrate was qualitatively monitored periodically by TLC analysis using silica gel plates (TLC Silica gel 60F 254-Aluminum sheets $20 \mathrm{~cm} \times 20 \mathrm{~cm}$, Merck KGaA, Darmstadt, Germany). Acetone:toluene (50:50 v:v) solvent mixture was used as the TLC solvent system, with the addition of few drops of glacial acetic acid and visualized under UV light and iodide staining. After confirming the completion, the enzymatic reaction was halted by filtering the immobilized lipase and molecular sieves from the reaction mixture and the acetone was removed by vacuum evaporation. The synthesized phenolipids were isolated by subjecting the crude product to silica gel column chromatography using acetone:toluene; 40:60 to 50:50. Preparative TLC was performed under the same conditions as above.

Figure 1. Esterification of $\mathrm{Q} 3 \mathrm{G}$ with acyl donor fatty acids. (a) Acetone, $3{ }^{\circ} \mathrm{A}$ molecular sieves, Candida antactica B lipase, $45^{\circ} \mathrm{C}$, Stirring, 24 h; R = Oleic acid, Stearic acid, Linoleic acid, $\alpha$-Linolenic, Eicosapentaenoic acid, and Docosahexaenoic acid.

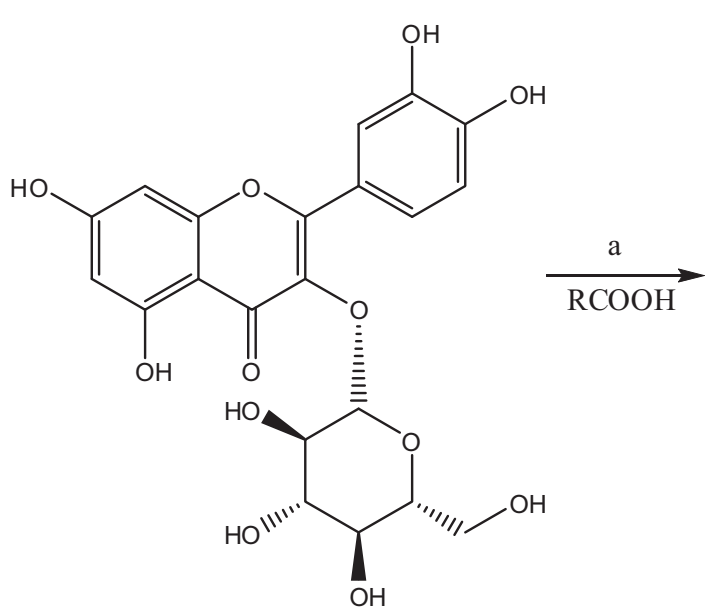

Quercetin-3-0-glucoside (Q3G)

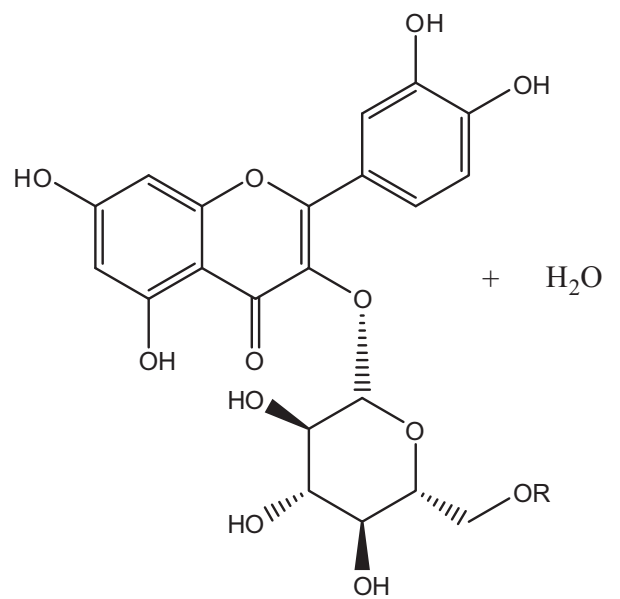

Fatty acid ester of Q3G

\subsection{Determination of Primary Oxidation in Bulk Fish Oil Model System}

Different concentrations of Q3G and fatty acid acylated derivatives of Q3G $\left(0.5,1,5\right.$, and $\left.10 \mathrm{mmol} \cdot \mathrm{L}^{-1}\right)$, dissolved in $1 \%$ dimethyl sulfoxide, were mixed with bulk fish oil and incubated at $40{ }^{\circ} \mathrm{C}$ for 3 and 
5 days. The lipid peroxides which were formed during lipid oxidation were determined as the peroxide value using acetic acid-chloroform method [15]. Briefly, oxidized fish oil was dissolved in 3:2 ratio of acetic acid-chloroform mixture and $0.5 \mathrm{~mL}$ of freshly prepared saturated KI solution was added and gently mixed. After $1 \mathrm{~min}, 30 \mathrm{~mL}$ of deionized water was added, followed by addition of $1 \mathrm{~mL}$ of starch. The liberated iodine was titrated with $0.1 \mathrm{~N}$ of $\mathrm{Na}_{2} \mathrm{~S}_{2} \mathrm{O}_{3}$. Percentage inhibition of lipid peroxidation was calculated, based on the peroxide values obtained.

\subsection{Preparation of Aqueous Emulsion Model System}

An aqueous emulsion (oil-in-water) of fish oil was prepared following a method described previously [16]. An emulsion of $10 \mathrm{mg}$ of fish oil per $1 \mathrm{~mL}$ of buffer $(\mathrm{pH} 7)$ containing $0.05 \mathrm{M}$ tris $\mathrm{HCl}, 0.15 \mathrm{M} \mathrm{KCl}$ and 4\% Tween 20 as an emulsifier, was prepared by homogenizing the mixture using a homogenizer (model PCU, Polytron ${ }^{\circledR}$, Luzernerstrasse, Littau-Luzern, Switzerland), at 4.5 speed for $30 \mathrm{~s}$.

\subsection{Determination of Primary Oxidation in Aqueous Emulsion Model System}

The procedure for ferric thiocyanate test was followed [16]. Ethanolic solutions (95\% ethanol) of Q3G and its esters in $0.5,1,5$, and $10 \mathrm{mmol} \cdot \mathrm{L}^{-1}$ concentrations were prepared in disposable $13 \times 100 \mathrm{~mm}$ borosilicate glass tubes and the solvent was completely evaporated under nitrogen flow. After solubilising the dried compounds with $10 \mu \mathrm{L}$ ethanol, $80 \mu \mathrm{L}$ of emulsion was added and vortexed. Oxidation was induced by adding $10 \mu \mathrm{L}$ of peroxyl radical generator, AAPH and incubated at room temperature for $40 \mathrm{~min}$. At the end of the incubation, further oxidation was halted immediately by adding $10 \mu \mathrm{L}$ of $1000 \mathrm{mg} \cdot \mathrm{L}^{-1}$ BHT into all the samples. Samples $(30 \mu \mathrm{L})$ were diluted with $210 \mu \mathrm{L}$ of $75 \%$ ethanol and $30 \mu \mathrm{L}$ of $3 \% \mathrm{NH}_{4} \mathrm{SCN}$ was added. After $3 \mathrm{~min}, 30 \mu \mathrm{L}$ of $2 \mathrm{mmol} \cdot \mathrm{L}^{-1}$ ferric chloride in $3.5 \% \mathrm{HCl}$ was added and absorbance was measured at $495 \mathrm{~nm}$ in 96-well microplates, using FLUOstar OPTIMA microplate reader (BMG Labtech, Durham, NC, USA). Blank samples were prepared for all the concentration levels of all the compounds, with no addition of emulsion, in order to eliminate the absorbance contributed by compounds themselves and blank absorbance values were subtracted from the sample absorbance. Percentage inhibition of lipid oxidation was calculated. The hydroperoxides, formed during lipid oxidation, react with ferrous ions to produce ferric ions, which are detected as the ferric thiocyanate red chromogen.

\subsection{Determining the Inhibition of Secondary Oxidation}

Secondary lipid oxidation, in both bulk fish oil and aqueous emulsion (oil-in-water) model systems, were determined by thiobarbituric acid reactive substances (TBARS) assay [16,17]. Desired concentrations of Q3G and fatty acid derivatives of Q3G in $95 \%$ ethanol $\left(0.5,1,5\right.$, and $\left.10 \mathrm{mmol} \cdot \mathrm{L}^{-1}\right)$ were placed in disposable $13 \times 100 \mathrm{~mm}$ borosilicate glass tubes and the solvent was completely evaporated under nitrogen flow. Twenty microliters of ethanol and $80 \mu \mathrm{L}$ of bulk fish oil or aqueous emulsion were added and vortexed well to ensure complete dispersion of the compounds in the bulk fish oil or aqueous emulsions. Induction of oxidation was achieved by heating the glass tubes, covered with breathable caps, for $3 \mathrm{~h}$ at $50{ }^{\circ} \mathrm{C}$ in a shaking incubator (model Apollo HP50, CLP Tools, San Diego, CA, USA).

The TBA reagent $(0.375 \%$ TBA and $15 \%$ TCA in $0.25 \mathrm{M} \mathrm{HCl})$ was added to all the oxidized fish oil or emulsion samples as well as the controls. After vortexing, all the samples were capped and placed in 
a water bath (Isotemp 205, Fisher scientific, Ubuque, IA, USA) at $80^{\circ} \mathrm{C}$ for $15 \mathrm{~min}$. The sample tubes were allowed to cool to room temperature and $2 \mathrm{~mL}$ of 1-butanol was added to each sample, followed by vortexing and centrifuging (IEC International Centrifuge, MA, USA) at 40× $\mathrm{g}$ for $20 \mathrm{~min}$. After loading the supernatant (1-butanol layer) into 96-well microplates, fluorescence intensity (FI) was measured for excitation at $532 \mathrm{~nm}$ and emission at 580, using FLUOstar OPTIMA plate reader (BMG Labtech). The percentage inhibition of the samples was calculated in comparison to controls with no antioxidants, using the equation below:

$$
\% \text { Inhibition }=100(\text { FI control }- \text { FI sample }) / F I \text { control }
$$

\subsection{LDL Oxidation}

\subsubsection{LDL Preparation}

The assay was conducted as described previously [18]. Briefly, prior to dialysis of LDL, cellulose dialysis tubing (Thermo Fisher Scientific Inc., Ottawa, ON, Canada) was pretreated to remove inherent antioxidants. The tubing was cut into the desired length $(6-8 \mathrm{~cm})$, soaked in deionized water for $15 \mathrm{~min}$ and heated at $80{ }^{\circ} \mathrm{C}$ in a $10 \mathrm{mmol} \cdot \mathrm{L}^{-1}$ sodium bicarbonate solution for $30 \mathrm{~min}$ while stirring. It was transferred into a $10 \mathrm{mmol} \cdot \mathrm{L}^{-1} \mathrm{Na} 2 \mathrm{EDTA}$ solution and soaked for $30 \mathrm{~min}$. Again, the tubing was transferred to the deionized water at $80{ }^{\circ} \mathrm{C}$ and soaked for $30 \mathrm{~min}$ while stirring. The membrane was cooled and submerged in 50\% ethanol solution and refrigerated. To perform the dialysis, the cellulose tubing was washed both inside and outside first with deionized water and then with $10 \mathrm{mmol} \cdot \mathrm{L}^{-1} \mathrm{PBS}$

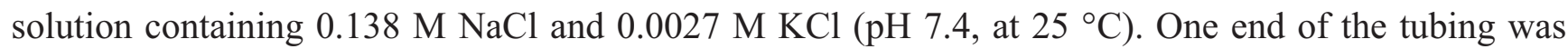
sealed, LDL was then injected into the open end which was then sealed completely. The sealed tubing was then immersed in $10 \mathrm{mmol} \cdot \mathrm{L}^{-1} \mathrm{PBS}$ solution at $4{ }^{\circ} \mathrm{C}$ for $24 \mathrm{~h}$. Buffer was changed every six hours. Once the dialysis was completed, the dialyzed LDL was immediately transferred into an amber colored vial and stored at $-80{ }^{\circ} \mathrm{C}$ for use within two weeks.

\subsubsection{Determination of Protein Content of Dialysed LDL}

Protein content of the dialyzed LDL was determined using the Lowry's method [19]. Bovine serum albumin was used as the standard protein source. Lowry's reagent $(1 \mathrm{~mL})$ was added to the blank, standards and dialyzed LDL and left for $20 \mathrm{~min}$. Then, Folin Ciocalteu reagent $(0.5 \mathrm{~mL})$ was added, followed by immediate thorough mixing and allowed to stand for $30 \mathrm{~min}$. Absorbance was measured at $750 \mathrm{~nm}$. Protein content of the LDL sample was calculated using the calibration curve of BSA (10, 20, 40 and $50 \mu \mathrm{g} / \mathrm{mL}$ ). The LDL sample was diluted appropriately with PBS to reach $100 \mu \mathrm{g} / \mathrm{mL}$ protein content.

\subsubsection{Inhibition of LDL Oxidation}

Oxidation was induced by two methods: using copper(II) sulfate and peroxyl radical generator, AAPH. Different concentrations $\left(1,10,100\right.$, and $\left.500 \mu \mathrm{mol} \cdot \mathrm{L}^{-1}\right)$ of Q3G derivatives and Q3G were prepared in methanol. Dialyzed LDL $(160 \mu \mathrm{L})$ was mixed with $20 \mu \mathrm{L}$ of the test compound in borosilicate glass tubes and $20 \mu \mathrm{L}$ of $\mathrm{Cu}^{2+}(10 \mu \mathrm{M}) / \mathrm{AAPH}(5 \mathrm{mM})$ was added. All the samples were incubated at $37{ }^{\circ} \mathrm{C}$ for $4 \mathrm{~h}$ in a shaking water bath. Solutions of $1 \mathrm{mmol} \cdot \mathrm{L}^{-1}$ EDTA and BHT were added to 
terminate oxidation in $\mathrm{Cu}^{2+}$ and peroxy radical-induced systems, respectively. LDL oxidation was determined using the TBARS assay [20].

\subsection{Statistical Analysis}

The statistical analysis was carried out using SAS 9.2 and Minitab 16 statistical software packages. The effect of the two factors: "type of test compound" and "concentration", on percentage inhibition of lipid oxidation was determined using a two factor factorial design of three replicates. The factor, "type of test compound", contained six levels (six different test compounds) and the factor "concentration" contained four levels (four different concentrations). The data was analyzed by ANOVA using general linear model. Multiple means comparison was carried out by least square means to identify the significant differences across the test compounds for each concentration. When there are no significant interactions between the two factors; "type of test compound" and "concentration", the significant differences among the effects of "type of test compound" were determined regardless of the concentration, using Tukey's multiple means comparison method. A probability of $p \leq 0.05$ was taken as statistically significant.

\section{Results}

\subsection{Inhibition of Oxidation in Bulk Fish Oil and Aqueous Emulsion}

In n-3 PUFA-rich bulk fish oil, the parent compound Q3G showed the highest percentage inhibition of primary oxidation over all the fatty acid derivatives of Q3G at $10 \mathrm{mmol} \cdot \mathrm{L}^{-1}$. However, the ALA, EPA and DHA derivatives of Q3G demonstrated considerable inhibition of 30\% to $40 \%$ (Table 1). When the secondary oxidation products in the bulk fish oil system was measured, all the tested compounds, except for the EPA and DHA derivatives of Q3G, demonstrated a concentration dependent effect where the higher concentrations provided the higher percent inhibition. Q3G esterified to LNA exhibited the highest percentage inhibition of $70 \%$ at the concentration of $10 \mathrm{mmol} \cdot \mathrm{L}^{-1}$ (Table 2). In general, the synthesized phenolipids had no significant $(p \leq 0.05)$ effect, compared to their parent flavonoid Q3G, on inhibition of both primary and secondary oxidation in bulk fish oil model system.

In primary lipid oxidation in oil-in-water emulsion (Table 3), all the six esters of Q3G exhibited better antioxidant properties than the parent compound Q3G, showing more than $50 \%$ inhibition in two or more tested concentration levels in the range of $0.5-10 \mathrm{mmol} \cdot \mathrm{L}^{-1}$. The STA, OLA and LNA derivatives of Q3G were effective, with more than 50\% inhibition in all the tested concentrations, showing that they are useful as antioxidants, even at low concentration levels such as $0.5 \mathrm{mmol} \cdot \mathrm{L}^{-1}$. More than $40 \%$ inhibition was observed in the EPA and DHA derivatives in all the tested concentrations and DHA derivative achieved $100 \%$ inhibition at $10 \mathrm{mmol} \cdot \mathrm{L}^{-1}$. In secondary oxidation of oil-in-water emulsion, all the phenolipids had their highest inhibition at $10 \mathrm{mmol} \cdot \mathrm{L}^{-1}$ and there was more than $30 \%$ inhibition (Table 3). Overall, the results showed that in the oil-in-water emulsion, Q3G stearate showed the greatest antioxidant protection since it reached the greatest inhibition at $5 \mathrm{mmol} \cdot \mathrm{L}^{-1}$ in the primary oxidation. In the case of secondary oxidation, Q3G stearate is the best antioxidant at $10 \mathrm{mmol} \cdot \mathrm{L}^{-1}$. The parent compound Q3G showed lower inhibition than the phenolipids, even at its most effective concentration level of $10 \mathrm{mmol} \cdot \mathrm{L}^{-1}$. It can be inferred that in the oil-in-water emulsion system, the phenolipids prepared by esterification of the fatty acids and Q3G have a better antioxidant activity than Q3G. 
Table 1. Inhibition of primary lipid oxidation in bulk fish oil in relation to concentration of the test compounds.

\begin{tabular}{|c|c|c|c|c|c|c|c|c|}
\hline \multirow{4}{*}{ Compound } & \multicolumn{8}{|c|}{ \% Inhibition (Relative to the Control) } \\
\hline & \multicolumn{4}{|c|}{ Day 3} & \multicolumn{4}{|c|}{ Day 5} \\
\hline & \multicolumn{4}{|c|}{ Concentration $\left(\mathrm{mmol} \cdot \mathrm{L}^{-1}\right)$} & \multicolumn{4}{|c|}{ Concentration $\left(\mathrm{mmol} \cdot \mathrm{L}^{-1}\right)$} \\
\hline & 0.5 & 1 & 5 & 10 & 0.5 & 1 & 5 & 10 \\
\hline Q3G & $0 \pm 0.0 \mathbf{c}$ & $0 \pm 0.0 \mathbf{c}$ & $45 \pm 7.1 \mathbf{b c}$ & $57 \pm 8.7 \mathbf{a}$ & $8 \pm 1.5 \mathrm{D}$ & $12 \pm 1.4 \mathrm{CD}$ & $34 \pm 4.4 \mathrm{BC}$ & $52 \pm 6.3 \mathrm{~A}$ \\
\hline Q3G stearate & $0 \pm 0.0 \mathbf{c}$ & $0 \pm 0.0 \mathbf{c}$ & $24 \pm 2.0 \mathbf{b c}$ & $26 \pm 4.0 \mathrm{bc}$ & $13 \pm 6.0 \mathrm{CD}$ & $22 \pm 2.6 \mathbf{C D}$ & $34 \pm 2.3 \mathbf{B C}$ & $29 \pm 3.2 \mathrm{BC}$ \\
\hline Q3G oleate & $7 \pm 2.8 \mathbf{c}$ & $0 \pm 0.0 \mathbf{c}$ & $14 \pm 6.5 \mathrm{c}$ & $12 \pm 9.3 \mathbf{c}$ & $0 \pm 0.0 \mathbf{D}$ & $6 \pm 3.8 \mathrm{CD}$ & $26 \pm 6.7 \mathrm{CD}$ & $30 \pm 15.2 \mathbf{B C}$ \\
\hline Q3G linoleate & $18 \pm 8.4 \mathbf{b c}$ & $16 \pm 13.4 \mathbf{b c}$ & $14 \pm 0.2 \mathrm{c}$ & $21 \pm 2.7 \mathbf{b c}$ & $11 \pm 1.0 \mathbf{C D}$ & $22 \pm 3.4 \mathbf{C D}$ & $28 \pm 3.0 \mathrm{C}$ & $26 \pm 5.6 \mathrm{C}$ \\
\hline Q3G $\alpha$-linolenate & $14 \pm 8.2 \mathrm{c}$ & $5 \pm 3.5 \mathrm{c}$ & $12 \pm 6.4 \mathrm{c}$ & $17 \pm 3.4 \mathbf{b c}$ & $5 \pm 3.5 \mathrm{D}$ & $11 \pm 4.8 \mathrm{CD}$ & $22 \pm 4.1 \mathbf{C D}$ & $40 \pm 2.7 \mathbf{A B}$ \\
\hline Q3G eicosapentaenoate & $18 \pm 7.5 \mathbf{b c}$ & $21 \pm 4.7 \mathbf{b c}$ & $34 \pm 3.8 \mathbf{b}$ & $30 \pm 5.0 \mathrm{bc}$ & $16 \pm 2.6 \mathrm{CD}$ & $18 \pm 0.5 \mathbf{C D}$ & $37 \pm 2.1 \mathbf{B}$ & $34 \pm 1.6 \mathbf{B C}$ \\
\hline Q3G docosahexaenoate & $9 \pm 7.0 \mathbf{c}$ & $17 \pm 1.0 \mathbf{b c}$ & $26 \pm 0.8 \mathbf{b c}$ & $21 \pm 1.2 \mathbf{b c}$ & $19 \pm 2.1 \mathbf{C D}$ & $21 \pm 6.2 \mathrm{CD}$ & $30 \pm 8.8 \mathbf{B C}$ & $38 \pm 1.3 \mathbf{A B}$ \\
\hline
\end{tabular}

Different concentrations of Q3G and fatty acid acylated derivatives of Q3G were incorporated into bulk fish oil and incubated at $40{ }^{\circ} \mathrm{C}$ for 3 and 5 days. The lipid peroxides formed were determined using acetic acid-chloroform method and \% inhibition of lipid peroxidation was presented as mean \pm standard deviation. Data from day 3 and day 5 were statistically analyzed separately. Different letters (a,b,c... and A,B,C...) denote significant differences among test compounds and concentrations, for day 3 and day 5 , respectively $(p \leq 0.05)$.

Table 2. Inhibition of secondary lipid oxidation in bulk fish oil in relation to the concentration of the test compounds.

\begin{tabular}{ccccc}
\hline \multirow{2}{*}{ Compound } & \multicolumn{4}{c}{ \% Inhibition (Relative to the Control) } \\
\cline { 2 - 5 } & \multicolumn{4}{c}{ Concentration $\left(\mathbf{m m o l} \cdot \mathbf{L}^{-\mathbf{1}}\right)$} \\
\hline & $\mathbf{0 . 5}$ & $\mathbf{1}$ & $\mathbf{5}$ & $\mathbf{1 0}$ \\
Q3G & $24 \pm 5.8 \mathbf{e}$ & $35 \pm 2.4 \mathbf{d}$ & $60 \pm 2.5 \mathbf{b}$ & $69 \pm 0.1 \mathbf{a}$ \\
Q3G stearate & $39 \pm 4.4 \mathbf{d}$ & $35 \pm 1.2 \mathbf{d}$ & $46 \pm 1.2 \mathbf{c d}$ & $52 \pm 2.1 \mathbf{b c}$ \\
Q3G oleate & $40 \pm 1.4 \mathbf{c d}$ & $60 \pm 0.7 \mathbf{b}$ & $64 \pm 1.5 \mathbf{a b}$ & $67 \pm 1.7 \mathbf{a b}$ \\
Q3G linoleate & $34 \pm 3.0 \mathbf{d}$ & $49 \pm 3.6 \mathbf{c}$ & $63 \pm 1.6 \mathbf{a b}$ & $70 \pm 0.6 \mathbf{a}$ \\
Q3G $\alpha$-linolenate & $38 \pm 1.0 \mathbf{d}$ & $33 \pm 4.4 \mathbf{d e}$ & $42 \pm 0.6 \mathbf{c d}$ & $51 \pm 0.9 \mathbf{b c}$ \\
Q3G eicosapentaenoate & $46 \pm 2.6 \mathbf{c d}$ & $40 \pm 1.9 \mathbf{c d}$ & $50 \pm 4.1 \mathbf{b c}$ & $31 \pm 1.1 \mathbf{d e}$ \\
Q3G docosahexaenoate & $50 \pm 4.2 \mathbf{c d}$ & $50 \pm 3.5 \mathbf{b c}$ & $47 \pm 6.4 \mathbf{c d}$ & $33 \pm 2.5 \mathbf{d e}$ \\
\hline
\end{tabular}

Induction of secondary oxidation in bulk fish oil was achieved by incubating for $3 \mathrm{~h}$ at $50{ }^{\circ} \mathrm{C}$ in a shaking incubator and oxidation was determined by TBARS assay. Data were presented as mean \pm standard deviation. Different letters $(\mathrm{a}, \mathrm{b}, \mathrm{c} \ldots)$ denote significant differences among test compounds for all concentrations $(p \leq 0.05)$. 
Table 3. Inhibition of lipid oxidation in oil-in-water emulsion in relation to the concentration of the test compounds.

\begin{tabular}{|c|c|c|c|c|c|c|c|c|}
\hline \multirow{4}{*}{ Compound } & \multicolumn{8}{|c|}{ \% Inhibition (Relative to the Control) } \\
\hline & \multicolumn{4}{|c|}{ Primary Oxidation } & \multicolumn{4}{|c|}{ Secondary Oxidation } \\
\hline & \multicolumn{4}{|c|}{ Concentration $\left(\mathrm{mmol} \cdot \mathrm{L}^{-1}\right)$} & \multicolumn{4}{|c|}{ Concentration $\left(\mathrm{mmol} \cdot \mathrm{L}^{-1}\right)$} \\
\hline & 0.5 & 1 & 5 & 10 & 0.5 & 1 & 5 & 10 \\
\hline Q3G & $29 \pm 6.7 \mathrm{c}$ & $39 \pm 9.4 \mathbf{b c}$ & $42 \pm 9.1 \mathbf{b c}$ & $18 \pm 15.3 \mathbf{c}$ & $0 \pm 0.0 \mathbf{F}$ & $0 \pm 0.0 \mathbf{F}$ & $9 \pm 2.3 \mathbf{E F}$ & $25 \pm 1.0 \mathbf{D E}$ \\
\hline Q3G stearate & $55 \pm 6.1 \mathbf{b c}$ & $74 \pm 7.1 \mathbf{a b}$ & $100 \pm 16.9 \mathbf{a}$ & $100 \pm 3.6 \mathbf{a}$ & $16 \pm 2.5 \mathbf{E}$ & $20 \pm 5.2 \mathbf{D E}$ & $69 \pm 2.3 \mathbf{A B}$ & $78 \pm 3.1 \mathbf{A}$ \\
\hline Q3G oleate & $53 \pm 8.5 \mathbf{b c}$ & $56 \pm 8.8 \mathbf{b c}$ & $55 \pm 4.1 \mathbf{b c}$ & $63 \pm 6.0 \mathrm{bc}$ & $11 \pm 3.4 \mathbf{E F}$ & $30 \pm 4.1 \mathbf{D}$ & $43 \pm 9.7$ CD & $49 \pm 14.4 C$ \\
\hline Q3G linoleate & $47 \pm 2.2 \mathbf{b c}$ & $66 \pm 0.6 \mathbf{b}$ & $60 \pm 8.1 \mathbf{b c}$ & $73 \pm 4.0 \mathbf{a b}$ & $0 \pm 0.0 \mathbf{F}$ & $3 \pm 1.0 \mathbf{F}$ & $45 \pm 2.0 \mathbf{C D}$ & $67 \pm 0.3 \mathbf{A B}$ \\
\hline Q3G $\alpha$-linolenate & $13 \pm 6.7 \mathbf{b c}$ & $15 \pm 0.1 \mathrm{c}$ & $34 \pm 4.0 \mathrm{c}$ & $64 \pm 3.0 c$ & $12 \pm 1.4 \mathbf{E F}$ & $24 \pm 6.5 \mathrm{DE}$ & $51 \pm 1.3 \mathbf{B C}$ & $63 \pm 0.6 \mathbf{B}$ \\
\hline Q3G eicosapentaenoate & $42 \pm 3.2 \mathbf{b c}$ & $56 \pm 5.4 \mathrm{bc}$ & $63 \pm 17.3 \mathbf{b c}$ & $65 \pm 12.2 \mathbf{b c}$ & $0 \pm 0.0 \mathbf{F}$ & $0 \pm 0.0 \mathbf{F}$ & $13 \pm 1.9 \mathbf{E F}$ & $32 \pm 1.8 \mathbf{D}$ \\
\hline Q3G docosahexaenoate & $49 \pm 5.0 \mathbf{b c}$ & $43 \pm 4.0 \mathbf{b c}$ & $54 \pm 19.3 \mathbf{b c}$ & $100 \pm 8.4 \mathbf{a}$ & $27 \pm 9.5 \mathrm{DE}$ & $26 \pm 3.8 \mathbf{D E}$ & $53 \pm 0.8 \mathbf{B C}$ & $58 \pm 8.0 \mathbf{B C}$ \\
\hline
\end{tabular}

Primary oxidation was induced by adding AAPH and incubated at room temperature for $40 \mathrm{~min}$ and oxidation was determined by ferric thiocyanate test. Induction of secondary oxidation in bulk fish oil was achieved by incubating for $3 \mathrm{~h}$ at $50{ }^{\circ} \mathrm{C}$ in a shaking incubator and oxidation was determined by TBARS assay. Data from primary and secondary oxidation were statistically analyzed separately and presented as mean \pm standard deviation. Different letters (a,b,c... and A,B,C...) denote significant differences among test compounds and concentrations, for \% inhibition of primary and secondary lipid oxidation, respectively $(p \leq 0.05)$.

Table 4. Inhibition of human LDL oxidation in vitro in relation to the concentration of the test compounds.

\begin{tabular}{|c|c|c|c|c|c|c|c|c|}
\hline \multirow{4}{*}{ Compound } & \multicolumn{8}{|c|}{$\%$ Inhibition (Relative to the Control) } \\
\hline & \multirow{2}{*}{\multicolumn{4}{|c|}{$\begin{array}{c}\mathrm{Cu}^{2+} \text {-Induction } \\
\text { Concentration }\left(\mu \mathrm{mol} \cdot \mathrm{L}^{-1}\right)\end{array}$}} & \multirow{2}{*}{\multicolumn{4}{|c|}{$\begin{array}{c}\text { AAPH Derived Peroxyl Radical-Induction } \\
\text { Concentration }\left(\mu \mathrm{mol} \cdot \mathrm{L}^{-1}\right)\end{array}$}} \\
\hline & & & & & & & & \\
\hline & 1 & 10 & 100 & 500 & 1 & 10 & 100 & 500 \\
\hline Q3G & $7 \pm 4.5 \mathrm{c}$ & $20 \pm 9.2 \mathbf{b c}$ & $20 \pm 1.8 \mathbf{b c}$ & $33 \pm 8.6 \mathbf{a b}$ & $10 \pm 2.6 \mathbf{C}$ & $10 \pm 2.7 \mathrm{C}$ & $16 \pm 7.2 \mathrm{C}$ & $29 \pm 2.0 \mathrm{C}$ \\
\hline Q3G stearate & $4 \pm 9.4 c$ & $14 \pm 7.5 \mathbf{b c}$ & $21 \pm 8.6 \mathbf{b c}$ & $29 \pm 4.2 \mathbf{b}$ & $4 \pm 5.9 \mathrm{C}$ & $11 \pm 5.0 \mathrm{C}$ & $14 \pm 7.5 \mathrm{C}$ & $27 \pm 8.5 \mathrm{C}$ \\
\hline Q3G oleate & $8 \pm 7.4 \mathrm{c}$ & $18 \pm 5.8 \mathbf{b c}$ & $14 \pm 5.3 \mathrm{bc}$ & $28 \pm 2.8 \mathbf{b c}$ & $12 \pm 7.2 \mathrm{C}$ & $9 \pm 2.8 \mathrm{C}$ & $15 \pm 3.9 \mathrm{C}$ & $17 \pm 13.6 \mathrm{C}$ \\
\hline Q3G linoleate & $14 \pm 3.4 \mathrm{bc}$ & $27 \pm 7.8 \mathbf{b c}$ & $29 \pm 5.9 \mathbf{b}$ & $34 \pm 6.3 \mathbf{a b}$ & $5 \pm 4.7 \mathrm{C}$ & $15 \pm 7.8 \mathrm{C}$ & $23 \pm 5.6 \mathrm{C}$ & $25 \pm 5.4 \mathrm{C}$ \\
\hline Q3G $\alpha$-linolenate & $40 \pm 5.6 \mathbf{a b}$ & $34 \pm 5.1 \mathbf{a b}$ & $31 \pm 2.9 \mathbf{a b}$ & $31 \pm 3.5 \mathbf{a b}$ & $28 \pm 9.5 \mathbf{A B}$ & $23 \pm 4.3 \mathrm{AB}$ & $23 \pm 2.0 \mathrm{AB}$ & $34 \pm 6.3 \mathbf{A B}$ \\
\hline Q3G eicosapentaenoate & $20 \pm 8.8 \mathbf{b c}$ & $43 \pm 6.8 \mathbf{a b}$ & $45 \pm 3.1 \mathbf{a b}$ & $51 \pm 2.6 \mathbf{a}$ & $21 \pm 3.8 \mathbf{A}$ & $36 \pm 6.7 \mathbf{A}$ & $38 \pm 1.7 \mathbf{A}$ & $41 \pm 2.9 \mathbf{A}$ \\
\hline Q3G docosahexaenoate & $7 \pm 5.5 c$ & $14 \pm 7.9 \mathbf{b c}$ & $27 \pm 6.7 \mathbf{b c}$ & $43 \pm 1.4 \mathbf{a b}$ & $13 \pm 3.7 \mathbf{B C}$ & $9 \pm 8.1 \mathbf{B C}$ & $30 \pm 10.0 \mathrm{BC}$ & $27 \pm 8.1 \mathbf{B C}$ \\
\hline
\end{tabular}

Data from $\mathrm{Cu}^{2+}$ - and peroxyl radical-induced LDL oxidation were statistically analyzed separately and presented as means \pm standard deviation. Different letters (a,b,c... and $\mathrm{A}, \mathrm{B}, \mathrm{C} \ldots$ ) denote significant differences among test compounds and concentrations, for $\%$ inhibition of $\mathrm{Cu}^{2+}$ - and peroxy radical- induced oxidation, respectively $(p \leq 0.05)$. 


\subsection{Inhibition of Oxidation in Human LDL}

The ability of the compounds to inhibit the plasma LDL oxidation in vitro represents the antioxidant capacity of the compounds to deal with cellular oxidative stress. Over $40 \%$ inhibition for $\mathrm{Cu}^{2+}$-induced oxidation has been shown by the EPA derivative of Q3G at $10 \mu \mathrm{mol} \cdot \mathrm{L}^{-1}$ and the DHA derivative at $500 \mu \mathrm{mol} \cdot \mathrm{L}^{-1}$ (Table 4). For the AAPH derived peroxyl radical-induced oxidation, the selected concentration range did not demonstrate a dose dependent effect, but the EPA and ALA derivatives were more effective than $\mathrm{Q} 3 \mathrm{G}$ in protecting the plasma LDL from oxidation. EPA derivative of Q3G, however, showed the highest inhibition in both experiments at concentrations of $500 \mu \mathrm{mol} \cdot \mathrm{L}^{-1}$.

\section{Discussion}

Presently, there is a growing interest in plant polyphenols, especially flavonoids, which can be applied as natural antioxidants in foods, pharmaceuticals and cosmetics [21]. Their application can be further enhanced by modifying the flavonoid structure by esterification [7]. In this study, Q3G was successfully acylated with six different long chain fatty acids $\left(\mathrm{C}_{18}\right.$ to $\left.\mathrm{C}_{22}\right)$ by lipase-catalyzed esterification. In all the reactions, the primary hydroxyl of the glycosyl group of the Q3G molecule was esterified with long chain fatty acids having various degrees of unsaturation (unsaturated 18:0, mono- 18:1 and poly-unsaturated $18: 2 ; 18: 3 ; 20: 5 ; 22: 6$ ). The esterification of flavonoid compounds, using Candida antarctica B lipase as a very effective biocatalyst for various acyl donors, is widely reported in the literature. Naringin [22], chlorogenic acid [23], rutin [8], quercetin [14], Q3G [10], hesperidin [24] and esculin [13] are some of the extensively used flavonoids in esterification studies, with different acyl donors, such as palmitic acid, OLA, STA, lauric acid, myristic acid, $\gamma$-linolenic acid and hexadecanedioic acid.

However, the antioxidant ability of the flavonoids is highly dependent on their chemical structure; type, position and number of the functional groups, and they behave differently in different media [16]. In the literature, lipophilicity has demonstrated its effect on the antioxidant activity in different ways. Improved lipophilicity of an antioxidant molecule could result an enhanced, a similar or a decreased activity compared to that of parent compound. The alkyl esters of caffeic acid displayed enhanced antiradical activity, while dihydrocaffeic acid showed a decreased effect [25]. Lipophilisation of rutin molecule using fatty acids had no effect on radical scavenging capacity of rutin [26]. Further, it can be explained that accessibility of hydroxyl groups to the free radicals is obstructed by the steric hindrance created by long acyl chains of fatty acids and it decreases the radical scavenging ability of the synthesized phenolipids. Moreover, the considerably different reaction conditions used in the antioxidant assays make it difficult to draw unifying conclusions [27].

Oxidation stability is a parameter with greater attention and significance, in terms of evaluating the quality of lipid based foods, which are highly prone to oxidative deterioration. In the present study, the effects of enhanced lipophilicity of these synthesized phenolipids on inhibition of lipid oxidation were determined using two lipid based food model systems of PUFA-rich fish oil; bulk oil and oil-in-water emulsion. In oil-in-water emulsion systems, the phenolipids acted as more effective antioxidants than their parent Q3G. However, Q3G acted as more effective antioxidant for the inhibition of oxidation in bulk oil. The polar paradox theory explains the behavior of antioxidants in different media; the lipophilic antioxidants are more effective in inhibiting oxidation in less lipophilic media and hydrophilic antioxidants 
are more effective in inhibiting oxidation in more lipophilic media [28,29]. This is further explained using the interfacial phenomenon; lipophilic antioxidants locate towards the oil-water interface in oil-in-water emulsions, the location where the oxidation is more prevalent, and thereby protect the oil phase from oxidation. Hydrophilic antioxidants move into the aqueous phase and this dilution makes them not sufficiently effective for protecting the oil phase [30]. In bulk oil systems, oxidation is believed to take place in the association colloids, formed by surface active molecules in the presence of a small quantity of water in bulk oil [31].

There are a number of previous studies, which have exhibited the effect of the lipophilicity of antioxidants in bulk oil and emulsion model systems. It has been found, using a series of alkyl ester derivatives of tyrosol and hydroxytyrosol, that the lipophilic esters are weaker antioxidants in bulk oil [32]. Further it was reviewed in [30] that esterification with long hydrocarbon chains changed the partitioning behavior of chlorogenic acid and increased its ability to concentrate at oil-water interface. Therefore, it is apparent that esterification improves the lipophilicity of the antioxidants and the lipophilic and hydrophilic antioxidants have different effectiveness in different media. According to the explanations used in the polar paradox theory, it is understood that change in lipophilicity of the antioxidant molecules changes their physical location in different food matrices, affecting the effectiveness of the antioxidants. The observations in the present study indicate that esterification with long chain hydrocarbons improved the lipophilicity of the Q3G which caused the Q3G ester to be orientated with the lipophilic long chain fatty acid embedded in the oil and the polar Q3G in the aqueous phase surrounding the oil droplet. This means that the polar Q3G remains at the oil-water interface which yields protection from the oxidation of the droplet.

It has been explained that the polar paradox theory is applicable only in certain concentration ranges since the effect of solubility starts dominating over the interfacial phenomenon [30]. Therefore, in lipophilic media, more lipophilic antioxidants are effective at the lower concentrations and more hydrophilic antioxidants are effective at their higher concentrations. In the present study, Q3G was not effective in lower concentrations in the bulk oil and it can be due to solubility effect, which is more dominating at the lower concentrations. Since, these phenolipids are more lipophilic than Q3G, they are soluble in the lipid matrix playing a dominating role in inhibiting oxidation. Further, Q3G was more effective at its higher concentrations, at which the interfacial phenomenon dominates over the solubility effect. Moreover, long chain fatty acids including n-3 and n-6 PUFA have been used in this study and are known to have numerous health advantages. Therefore, in addition to the protection from oxidation, these novel molecules may provide clinical advantages.

The observations in the LDL oxidation model, reporting greater inhibition by several phenolipids compared to the Q3G, are supported by the previous study [6], which demonstrated that ethyl esterification of phenolic acids made them better antioxidants in inhibiting LDL oxidation. The previous studies have demonstrated the use of polyphenols including flavonoids as the inhibitors of LDL oxidation [30,33,34]. Moreover, the beverages prepared from flavonoids-rich fruits, such as red wine and grape juice, have been found to inhibit the human LDL oxidation in vitro [35-37]. Our present study demonstrated that increasing lipophilicity of the Q3G makes them more potent antioxidants in inhibiting the LDL oxidation. In addition, it has been postulated that an increase in lipophilicity of the ethyl esters facilitates the incorporation of the antioxidant molecules through the lipid layer of the LDL particles [6]. 
To better understand the mechanisms which make the incorporating of these esters more feasible, further studies are needed.

\section{Conclusions}

It can be concluded that all the derivatives of Q3G showed more than 30\% inhibition in secondary oxidation and more than $50 \%$ inhibition in primary oxidation in the oil-in-water emulsion. The synthesized compounds were more effective in inhibiting both primary and secondary oxidation in oil-in-water emulsions than Q3G. However, Q3G reported 50\%-60\% inhibition at $10 \mathrm{mM}$ concentration in bulk oil. These modified compounds were not significantly effective in bulk oil system, when compared with Q3G $(p \leq 0.05)$. Therefore, these synthesized phenolipids have a potential for use as effective antioxidants in PUFA containing emulsion based foods such as mayonnaise, salad dressings, and sour cream. The EPA and DHA derivatives of Q3G exhibited more than $50 \%$ inhibition in $\mathrm{Cu}^{2+}$-induced LDL oxidation. The EPA and ALA derivatives were more effective in AAPH derived peroxyl radical-induced oxidation than Q3G $(p \leq 0.05)$. Therefore, these novel phenolipids can be introduced as potential drug candidates for pathogenic conditions, such as atherosclerosis and related diseases. Further research is required to understand the biological functions of the modified Q3G.

\section{Acknowledgments}

The authors acknowledge the funding provided by the Discovery Grant program of the Natural Sciences and Engineering Research Council (NSERC) of Canada and the Canada Research Chair program.

\section{Author Contributions}

Ziaullah and Sumudu N. Warnakulasuriya synthesized the novel esters of Q3G. Sumudu N. Warnakulasuriya performed all experiments and wrote the first draft of the manuscript. All of the authors discussed the results and contributed to the writing of the manuscript. H. P. Vasantha Rupasinghe is the Principal Investigator.

\section{Abbreviations}

$\begin{array}{ll}\text { STA } & \text { Stearic acid (18:0) } \\ \text { OLA } & \text { Oleic acid }(18: 1 \mathrm{n}-9) \\ \text { LNA } & \text { Linoleic acid }(18: 2 \mathrm{n}-6) \\ \text { ALA } & \text { Alpha-linolenic acid }(18: 3 \mathrm{n}-3) \\ \text { EPA } & \text { Eicosapentaenoic acid }(20: 5 \mathrm{n}-3) \\ \text { DHA } & \text { Docosahexaenoic acid (22:6n-3) } \\ \text { PUFA } & \text { Polyunsaturated fatty acid(s) } \\ \text { AAPH } & \text { 2,2'-Azobis(2-amidinopropane) dihydrochloride } \\ \text { BHT } & \text { Butylated hydroxytoluene } \\ \text { LDL } & \text { Low density lipoprotein } \\ \text { Q3G } & \text { Quercetin-3-O-glucoside } \\ \text { TBA } & \text { 2-Thiobarbituric acid } \\ \text { TBARS } & \text { Thiobarbituric acid reactive substances } \\ \text { TCA } & \text { Trichloroacetic acid }\end{array}$




\section{Conflicts of Interest}

The authors declare no conflict of interest.

\section{References}

1. Russo, G.L. Dietary n-6 and n-3 polyunsaturated fatty acids: From biochemistry to clinical implications in cardiovascular prevention. Biochem. Pharmacol. 2009, 77, 937-946.

2. Sun, Y.; Wang, W.; Chen, H.; Li, C. Autoxidation of unsaturated lipids in food emulsion. Crit. Rev. Food Sci. Nutr. 2011, 5, 453-466.

3. Kathirvel, P.; Rupasinghe, H.P.V. Plant-derived antioxidants as potential omega-3 PUFA stabilizers. In Fish Oil: Production, Consumption and Health Benefits; Dijk, M.V., Vitel, J., Eds.; Nova Science Publishers USA: Hauppauge, NY, USA, 2011; pp. 143-154.

4. Kanner, J. Dietary advance lipid oxidation end products are risk factors to human health. Mol. Nutr. Food Res. 2007, 51, 1094-1101.

5. Katsube, T.; Imawaka, N.; Kawano, Y.; Yamazaki, Y.; Shiwaku, L.; Yamane, Y. Antioxidant flavonol glycosides in mulberry (Morus alba L.) leaves isolated based on LDL antioxidant activity. Food Chem. 2006, 97, 25-31.

6. Chalas, J.; Claise, C.; Edeas, M.; Messaoudi, C.; Vergnes, L.; Abella, A.; Lindenbaum, A. Effect of ethyl esterification of phenolic acids on low-density lipoprotein oxidation. Biomed. Pharmacother. 2001, 55, 54-60.

7. Salem, J.H.; Chevalot, I.; Harscoat-Schiavo, C.; Paris, C.; Fick, M.; Humeau, C. Biological activities of flavonoids from Nitraria retusa (Forssk.) Asch. and their acylated derivatives. Food Chem. 2011, 124, 486-494.

8. Lue, B.M.; Guo, Z.; Xu, X. Effect of room temperature ionic liquid structure on the enzymatic acylation of flavonoids. Process Biochem. 2010, 45, 1375-1382.

9. Montenegro, L.; Carbone, C.; Maniscalco, C.; Lambusta, D.; Nicolosi, G.; Ventura, C.A.; Puglisi, G. In vitro evaluation of quercetin-3-O-acyl esters as topical prodrugs. Int. J. Pharm. 2007, 336, 257-262.

10. Mellou, F.; Lazari, D.; Skattsa, H.; Tselepis, A.D.; Kolisis, F.N.; Stamatis, H. Biocatalytic preparation of acylated derivatives of flavonoid glycosides enhances their antioxidant and antimicrobial activity. J. Biotechnol. 2005, 116, 295-304.

11. Laguerre, M.; Bayrasy, C.; Lecomte, J.; Chabi, B.; Decker, E.A.; Wrutniak-Cabello, C.; Cabello, G.; Villeneuve, P. How to boost antioxidants by lipophilization? Biochimie 2013, 95, 20-26.

12. Chebil, L.; Humeau, C.; Falcimaigne, A.; Engasser, J.M.; Ghoul, M. Review: Enzymatic acylation of flavonoids. Process Biochem. 2006, 41, 2237-2251.

13. Ardhaoui, M.; Falcimaigne, A.; Ognier, S.; Engasser, J.M.; Moussou, P.; Pauly, G.; Ghoul, M. Effect of acyl donor chain length and substitutions pattern on the enzymatic acylation of flavonoids. J. Biotechnol. 2004, 110, 256-271.

14. Ziaullah; Bhullar, K.S.; Warnakulasuriya, S.N.; Rupasinghe, H.P.V. Biocatalytic synthesis, structural elucidation, antioxidant capacity and tyrosinase inhibition activity of long chain fatty acid acylated derivatives of phloridzin and isoquercitrin. Bioorg. Med. Chem. 2013, 21, 684-692. 
15. Official Methods and Recommended Practices of the American Oil Chemists' Society Method Cd 8-53; Firestone, D., Ed.; American Oil Chemists' Society Champaign: Urbana, IL, USA, 1997.

16. Rupasinghe, H.P.V.; Erkan, N.; Yasmin, A. Antioxidant protection of eicosapentaenoic acid and fish oil oxidation by polyphenolic-enriched apple skin extract. J. Agric. Food Chem. 2010, 58, 1233-1239.

17. Huber, G.M.; Rupasinghe, H.P.V.; Shahidi, F. Inhibition of oxidation of omega-3 polyunsaturated fatty acids and fish oil by quercetin glycosides. Food Chem. 2009, 117, 290-295.

18. Thilakarathna, S.H.; Rupasinghe, H.P.V. Anti-atherosclerotic effects of fruit bioactive compounds: A review of current scientific evidence. Can. J. Plant Sci. 2012, 92, 407-419.

19. Lowry, O.H.; Rosebrough, N.J.; Farr, A.L.; Randall, R.J. Protein measurement with the Folin phenol reagent. J. Biol. Chem. 1951, 193, 165-275.

20. Xu, B.J.; Yuan, S.H.; Chang, S.K.C. Comparative studies on the antioxidant activities of nine common food legumes against copper induced LDL oxidation in vitro. J. Food Sci. 2007, 72, 522-527.

21. Danihelova, M.; Viskupičova, J.; Šturdika, E. Lipophilization of flavonoids for their food, therapeutic and cosmetic applications. Acta Chim. Slovaca 2012, 5, 59-69.

22. Passicos, E.; Santarelli, X.; Coulon, D. Regioselective acylation of flavonoids catalyzed by immobilized Candida antarctica lipase under reduced pressure. Biotechnol. Lett. 2004, 26, 1073-1076.

23. Lorentz, C.; Dulac, A.; Pencreach, G.; Ergan, F.; Richomme, P.; Soultani-Vigneron, S. Lipase-catalyzed synthesis of two new antioxidants: 4-O- and 3-O-palmitoyl chlorogenic acids. Biotechnol. Lett. 2010, 32, 1955-1960.

24. Chebil, L.; Anthoni, J.; Humeau, C.; Gerardin, C.; Engasser, J.M.; Goul, M. Enzymatic acylation of flavonoids: Effect of the nature of the substrate, origin of lipase and operating conditions on conversion yield and regioselectivity. J. Agric. Food Chem. 2007, 55, 9496-9502.

25. Silva, F.A.; Borges, F.; Guimarães, C.; Lima, J.L.; Matos, C.; Reis, S. Phenolic acids and derivatives: Studies on the relationship among structure, radical scavenging activity, and physicochemical parameters. J. Agric. Food Chem. 2000, 48, 2122-2126.

26. Viskupicova, J.; Danihelova, M.; Ondrejovic, M.; Liptaj, T.; Sturdik, E. Lipophilic rutin derivatives for antioxidant protection of oil-based foods. Food Chem. 2010, 123, 45-55.

27. Sharma, O.P.; Bhat, T.K. DPPH antioxidant assay revisited. Food Chem. 2009, 113, 1202-1205.

28. Porter, W.L. Recent trends in food applications of antioxidants. In Autoxidation in Food and Biological Systems; Simic, M.G., Karel, M., Eds.; Plenum Press: New York, NY, USA, 1980; pp. 295-365.

29. Frankel, E.N. Antioxidants in lipid foods and their impact on food quality. Food Chem. 1996, 57, $51-55$.

30. Shahidi, F.; Zhong, Y. Revisiting the polar paradox theory: A critical overview. J. Agric. Food Chem. 2011, 59, 3499-3504.

31. Chaiyasit, W.; Elias, R.J.; McClements, D.J.; Decker, E.A. Role of physical structures in bulk oils on lipid oxidation. Crit. Rev. Food Sci. Nutr. 2007, 47, 299-317.

32. Mateos, R.; Trujillo, M.; Pereira-Caro, G.; Madrona, A.; Cert, A.; Espartero, J.L. New lipophilic tyrosyl esters: Comparative antioxidant evaluation with hydroxytyrosyl esters. J. Agric. Food Chem.

2008, 56, 10960-10966. 
33. Frankel, E.N.; Kanner, J.; German, J.B.; Parks, E.; Kinsella, J.E. Inhibition of oxidation of human low-density lipoprotein by phenolic substances in red wine. Lancet 1993, 341, 454-457.

34. Safari, M.; Sheikh, N. Effects of flavonoids on the susceptibility of low-density lipoprotein to oxidative modification. Prostaglandins Leukot. Essent. Fatty Acids 2003, 69, 73-77.

35. Thilakarathna, S.H.; Rupasinghe, H.P.V.; Needs, P.W. Apple peel bioactive rich extracts effectively inhibit in vitro human LDL oxidation. Food Chem. 2013, 138, 463-470.

36. Miyagi, Y.; Miwa, K.; Inoue, H. Inhibition of human low-density lipoprotein oxidation by flavonoids in red wine and grape juice. Am. J. Cardiol. 1997, 80, 1627-1631.

37. Kerry, N.; Abbey, M. Red wine and fractionated phenolic compounds prepared from red wine inhibit low density lipoprotein oxidation in vitro. Atherosclerosis 1997, 135, 93-102.

(C) 2014 by the authors; licensee MDPI, Basel, Switzerland. This article is an open access article distributed under the terms and conditions of the Creative Commons Attribution license (http://creativecommons.org/licenses/by/4.0/). 